\title{
The Approach of Space-Time Cube to Visualizing Movement Data
}

\author{
Hong Thi Nguyen
}

\begin{abstract}
The movement swops time for location because a moving object must take time to displace from a place to another. Basically, the movement data of a moving object consist of time, location, and attributes, which are recorded discretely as data tuples and may be structured as a data table. This paper resumes the approaches to visualizing movement data according to two strategies, spatio-temporal data and multivariable spatio-temporal data, based on the concept of time geography. A table of spatio-temporal data which comprises the fields of time and location may be represented on a space-time cube. A table of multivariable spatio-temporal data which consists of the fields of time, location, and attributes may be divided small tables, where the table of spatio-temporal data is represented on a space-time cube, the tables of attibutes changing over time and/or at locations are represented as graphs to be integrated into the cube according to suitably shared variables. The movement data of a flying object including four variables, 2 variables indicating ground position, 1 variable indicating time, and 1 variable indicating elevation, are represented on a $4 \mathrm{D}$ cube.
\end{abstract}

Index Terms-Movement data, visualization, space-time cube, spatio-temporal data, moving object.

\section{INTRODUCTION}

The concept of time geography proposed by Hagerstrand in 1970 utilized a three-dimensional Cartesian coordinate system to indicate spatio-temporal positions of objects. In that, a spatio-temporal position of an object is indicated by a position on the Earth and a position of time. A space-time cube using three-dimensional Cartesian coordinate system may only show three variables, two variables indicating ground position, another indicating time. The representation of data of more three variables is still a challenge to authors.

This paper studies the applications of space-time cube for movement data, from spatio-temporal data to multivariable spatio-temporal data, then flying data. The data of a moving object are recorded discretely according to an appropriate method and structured as a data table. The paper studies the applications of space-time cube for representing spatial data associated with temporal data, and the modification of space-time cube to depict moving objects with multivariable spatio-temporal data. In addition, the approach of four-dimensional cube portraying the flying objects and its applications for monitoring the collision of two objects and planning new flying paths is also mentioned.

This article is structured as follows. The next section is the study of the components of movement dataset and classify

Manuscript received November 14, 2014; revised March 20, 2015

Hong Thi Nguyen is with University of Information Technology (UIT), Vietnam National University in Hochiminh City, Vietnam (e-mail: hongnguyen1611@gmail.com). them into spatio-temporal data and multivariable spatio-temporal data. In third section, the paper mentions the applications of space-time cube for displaying movements of spatio-temporal data. The fourth section resumes the applications of space-time cube for depicting moving objects of multivariable spatio-temporal data. The final section is the summary of the paper.

\section{The Components of A Set of Movement DatA}

An object is a spatially homogenous entity having particular locations and separate boundaries. An object is represented as a geometrical point indicated by a space position, a time position, and attributes. A set of movement data is structured as a table of data records and fields of space position, time position, and attributes. At a time point, an object occupies one and only one position in space, $f_{g}: t_{i} a g_{i}$, where $t_{i}$ is a time point, $t_{i} \in T, g_{i}$ is a ground position, $g_{i} \in G$. At a time point and at a location, an attribute has one and only one value, $f_{n}:\left(t_{i}, g_{i}\right) a a_{n}^{i}$, where $a_{n}^{i}$ is an value of an attribute $A_{n}, \quad n=1,2, . ., N$ with , at $\left(t_{i}, g_{i}\right)$.

For movement data, every object occupies one and only one position in space at a time point. The principle results in the relationship among object $o_{n}$, time $t$, and location $g$ in three inferences:

1) Inference 1:An object may be inferred from a time and a location : $\left(t_{i}, g_{i}\right) a o_{n}$.

2) Inference 2:A location may be inferred from a time and an object: $\left(t_{i}, o_{n}\right) a g_{i}$

3) Inference 3:The time point is not determined if the location of the object is known because the object may occupy a certain location at more one time point. The tuples $\left(o_{n}, t_{i}, g_{i}\right)$ and $\left(o_{n}, t_{j}, g_{i}\right)$ are defined that at the time point $t_{i}$ and $t_{j}$, the object $o_{n}$ locates at $g_{i}$ and $g_{j}$, respectively. If $g_{j_{1}}=. .=g_{j_{m}}=g_{i}$, where $j_{m}=i+m$, with $m=1,2, \ldots M$, then the object $o_{n}$ stays at the location $g_{i}$ from $t_{i}$ to $t_{i+M}$. If $j=i+K$ with $K \neq 1$, then the object $o_{n}$ returns back the location $g_{i}$ at $t_{i+K}$.

The result shows that the triad What - Where - When [1] and the triad Object - Location - Time [2] are defined completely in the inferences 1 and 2.

\section{The Visualization of SPatio-Temporal Data of MOVEMENTS}

\section{A. The Spatio-Temporal Data of a Moving Object}

A moving object is considered mathematically as a 
geometrical point that displaces continuously over time. The movement of an object on ground is depicted with a continuous path traced by the object on the Earth when it moves and called ground trajectory. Every point of the ground trajectory is a time-referenced ground position. Technically, the trajectory is represented as a poly-line time-ordered connecting a finite number of time-referenced ground positions. The data of ground positions associated with time positions are recorded discretely as the records of a data table including the field of time positions and two fields of coordinates of ground positions, also called the variable of time and the variables of location, respectively. These data records are made by recording the locations where the object passes along with the time point when the object comes, with the following methods [3]:

1) Time-based recording: the coordinates of ground positions of the object are recorded at defined time points or at regularly spaced time points.

2) Location-based recording: the data are recorded at defined locations or regularly spaced places.

3) Change-based recording: the locations along with time points are recorded when there is some change, e.g. the change of direction.

4) Event-based recording: the data of a record are made when an event happens.

5) Miscellaneous approach: the data records may be made by the combination of the above ways.

Each value of the time variable in the data table, termed time point or time position, is defined as a time interval of which the duration is determined so that at each time point, the object exists at one and only one ground position.

\section{B. The Visualization of Movement Data}

Basically, the movement converts time into location. At each time point, a moving object exists at one and only one ground position, but at a ground position, a moving object may exist many times at various time points. In the relationship between location and time of a moving object, the time is the reference variable while the location is a dependent variable. The time is a an important factor in considering movements. The movement data are technically recorded as data tuples of <location, time> and may be structured as a data table, where each time point associates with a ground position including two variables of the coordinate system. The movement of an object is depicted traditionally with its ground trajectory which is shown on a two-dimensional map with a ground-referenced coordinate system such as the geographic coordinate system indicated by longitudes and latitudes, or the world geodetic system in 1984 (WGS84), and so on. Each time point associated with a ground position forms a data record presented as a row of the data table. Geographic maps of various themes have been edited on two-dimensional coordinate system, published by many countries and institutions to depict visually the location of spatial entities as well as the relationships between them. Meanwhile, the variables of time and attributes are displayed on maps as symbols, text, or colors.

Traditionally, the data of locations are presented as a graph on an two-dimensional orthogonal coordinate system, of which each axis $x$ and $y$ represents a ground variable of the data table as a dimension. On a two-dimensional map, the movement of an object is shown as a path time-ordered connecting the places where the object passes, this path is termed ground trajectory. A well-known illustration of representing movement data is the 1812 Napoleon's march on Moscow created by Charles Joseph Minard in 1862 on a two-dimensional map with 6 types of data, the number of troops, distance, air temperature, geographic location of battlefields, direction of march, and dates relative to specific locations [4], [5]. In this illustration, the locations of battlefields are displayed on a map of the war area; the dates related to combats are noted as legends beside battlefields; the number of soldiers is represented with the thickness of the line showing the march and retreat; and the air temperature is shown on a two-dimensional coordinate system integrated into the war map as a legend under the retreat of the troops, where an axis indicates the retreat and another the corresponding temperatures.

The movement data of an object may be structured as a table of three variables, where two variables $x$ and $y$ represent ground position and the variable $t$ represents corresponding time point. The concept of time geography results in the utilization of a three-dimensional Cartesian coordinate system as a space-time cube, briefly STC, to depict the movement of objects [6]. In a space-time cube, the movement of an object is portrayed with a ground trajectory and a space-time path. The ground trajectory time-ordered connecting the ground positions where the object passes is made on a plane representing the Earth. The space-time path is a path time-ordered connecting points associating time positions with ground positions where the object passes. Kraak represented Napoleon's campaign in a space-time cube with the vertical time axis to display the space-time path of the operation. He utilized another three-dimensional cube to show the number of soldier at locations [7]. The directions of Napoleon's operation, march and retreat, are shown on the map at the bottom of space-time cube. The representation depicts Napoleon's operation line, march and retreat, the change of the number of troops at each battle. In Kraak's representation, five of six kinds of data of the campaign are displayed, the air temperature on the operation line and at battles is not considered. The illustration shows the loss of soldiers on the march and retreat to depict the defeat of the campaign, but it does not represent time- or location-referenced air temperature to enable human to analyze the cause of the defeat.

Several cities have been providing visitors with the information of city bus drawn on traditional two-dimensional maps. Basically, bus maps show densified networks of bus routes and bus stops. All buses of a route must run from the departure station to the end and visit all bus stops of the route. In reality, there are many buses take a bus route in a day, each of which is called a trip of the route. Each trip is indicated with the time of departure, the time of arrival, the times of reaching bus stops, and coded with a natural number as a trip number. The location-based recording is an approach suitable for constituting the data table of a route, where each data field is noted with a trip number, and each data record is noted with the time when the bus of the trip number visits a stop. Current bus maps only display routes, not show the information concerning trips such as departure times, arrival times, stop times at bus stops. Therefore, it is difficult for users to design a bus path convenient for their individual needs. 
In the temporal geography, a space-time cube comprises 2 axes indicating ground locations as a geographic map, and 1 axis indicating time. A space-time cube may be utilized to display not only routes but also trips of each route in a bus network. According to the approach, a bus route is displayed as a polyline time-ordered connecting bus stops from the departure station to the end station on a city map indicated by two axes of the space-time cube. Trips of the route are space-time paths time-ordered connecting the spatio-temporal points, each of which refers to a bus stop and the time point when the bus of the trip reaches the stop, indicated in the data table. The display screen may be densified by several trips on a bus route. The trips on a bus route may be normalized with a space-time path while the time bar on the time axis of the cube may be moved along the axis to indicate a specific trip. The space-time cube of visualizing bus trips is used to plan new bus routes in a city and may be also used to find a reasonable bus path by passengers (Fig. 1), [8], [9].

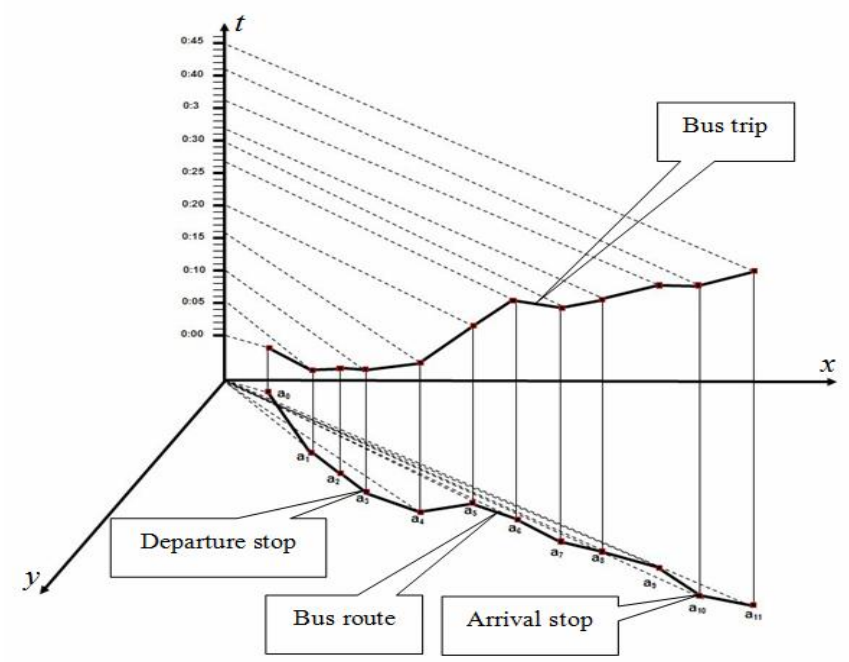

Fig. 1. A space-time cube representing bus route and bus trip (Source [8]).

\section{The Result}

The approach of space-time cube offers really a good method to display visually spatio-temporal data. For movement data that are recorded with the discretization of ground trajectories and space-time paths, a space-time cube represents the swop of time for location. Several authors have been try to develop many applications for representing spatio-temporal data of movements in various kinds of problems. In reality, moving objects own diverse attributes, some of which do not change, some change over time, others change at locations. The problems on view, display, interface have been solved by several authors with graphic techniques as abstraction, aggregation, show/hide, and so on [8], [10], [11]. The visualization of multivariable spatio-temporal data is also a challenge that is mentioned in the next section.

\section{The Visualization of Multivariable SPATIO-TEMPORAL DATA OF MOVEMENTS}

\section{A. The Multivariable Spatio-Temporal Data}

Generally, the movement data comprises a ordered sequence of time points, the locations referred to the time sequence, and attributes associated with time or location. The data of locations consists of two coordinates indicating the ground positions. The attributes of moving objects may change over time and/or at locations, e.g. the weight of a lorry can be change at a particular location during a time interval, the number of Napoleon's troops in 1812 campaign reduces after each combat, the speed of a vehicle change at some specific locations, and so on.

\section{B. The Cubes for Visualizing Multivariable Spatio-Temporal Data}

The display of attributes concurrent with data of time and location enables to analyze better data in different problems. Many authors have been proposing several solutions for this problem. In this sub-section, we revisit the approaches applying space-time cube for representing multivariable spatio-temporal data.

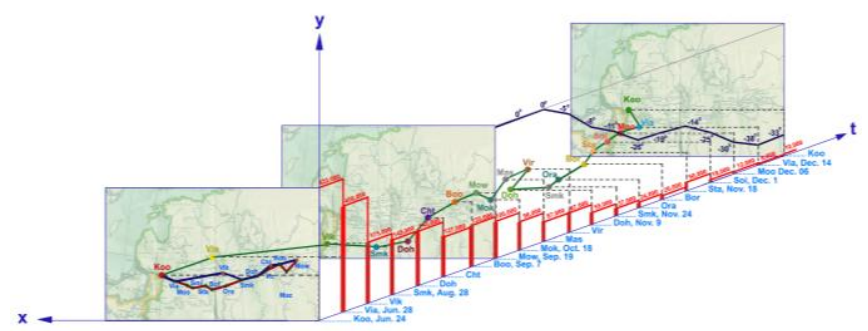

Fig. 2. A multivariable space-time cube for Napoleon's campaign (Source [12])

The concept of space-time cube is extended to represent multivariable spatio-temporal data, where a space-time cube is used to display spatial and temporal data and an attribute cube is formed by replacing the plane of locations with the plane of parallel coordinates to show attributes. This representation may portray the variation of each attribute over time in the attribute cube. It also offers the view on the relation between attributes at a time point, but the relationship between attributes and location is studied by mapping from attribute cube onto space-time cube [13].

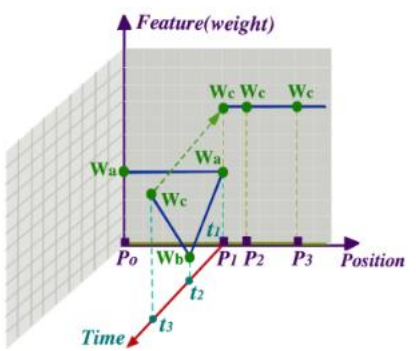

Fig. 3. An adaptive cube tracking the lorry movement (Source [14]).

Tran and Nguyen represented the 1812 Napoleon's campaign in a multivariable-space-time cube. The campaign is portrayed in a space-time cube with the horizontal time axis to display the space-time path of the operation, where the locations of battles are indicated on maps joined with the time axis at the corresponding days. The number of troops and air temperature are displayed as a time-referenced histogram and a curve along the time axis, respectively; the directions of the operation, march and retreat, are depicted as a ground trajectory on the map. The histogram of the number of soldiers enables viewer to perceive the great loss of the troops after each combat and along the operation path, as well as the correlation between the histogram and the temperature 
curve explain part of Napoleon's disastrous defeat in this campaign (Fig. 2), [12].

Several lorries need be monitored along road, not only their location over time but also goods they carries. A space-time cube can represent time-referenced locations of lorries, but it cannot display the weight of goods over time or at locations. Tran and Nguyen proposed an adaptive cube to depict the state of lorry, it shows time-referenced locations concurrently with location- and time-referenced weight. At a certain place, the lorry may stay in a time interval, during which the location does not change, but the weight may vary. At the place, a time axis of the adaptive cube is formed to show the change of weight (Fig. 3), [14].

\section{The Four-Dimensional Cube for Representing Flying Objects}

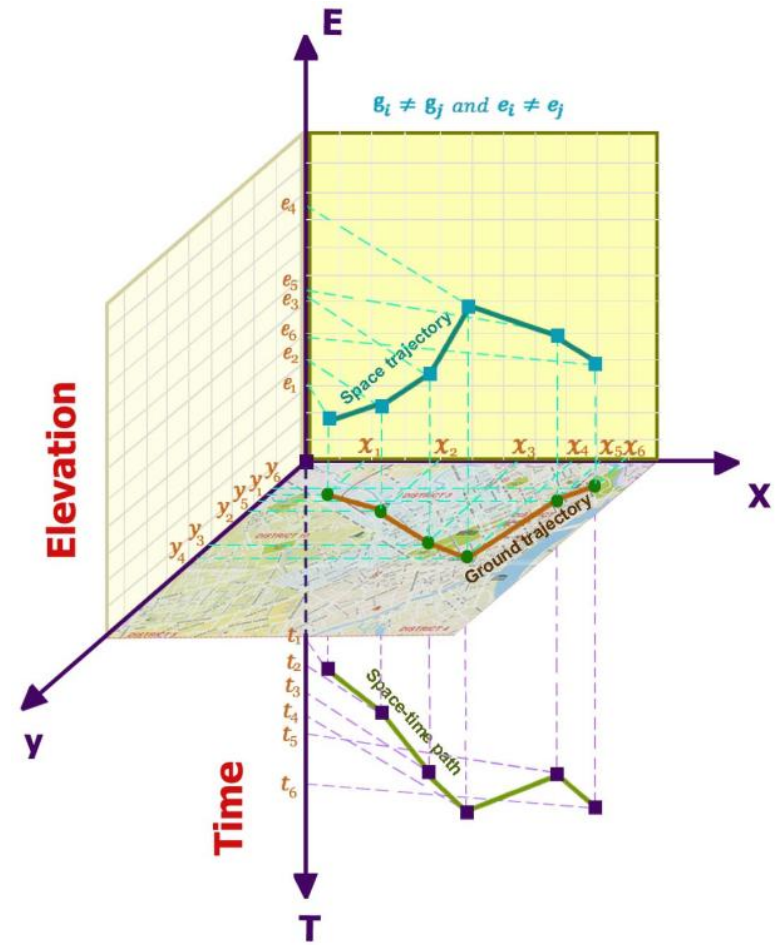

Fig. 4. A four-dimensional cube visualizing flying objects (Source [15]).

The movement of a flying object is depicted with a space trajectory. The location of a flying object is indicated with two dimensions on ground and one dimension of elevation. A four-dimensional cube is constituted by integrating a 3D cube into a space-time cube, where they share the plane indicating ground positions, the time axis of the space-time cube and the elevation axis of the 3D cube are inverse. The four-dimensional cube is utilized to estimate the possibility of collision of two flying objects and to plan a new flying path (Fig. 4), [15].

\section{CONCLUSION}

The paper approached temporal geography to review the author's works of using space-time cube to visualize movement data. The movement of an object is portrayed technically with a data table formed by recording discretely each data tuple of <time, location, attribute>, and shown as a ground trajectory and a space-time path. The approach of space-time cube is applied for representing bus routes, bus trips in a bus network to enable planners to plan city bus networks, as well as passengers to find the bus path appropriate to their individual needs. The paper also recaps the approach of multivariable space-time cube to portray movement data including location, time, and attributes. The applications of multivariable space-time cube for the data of Napoleon's campaign and monitoring lorries are considered as illustrations. The applications of four-dimensional cube for flying object are also mentioned. In summary, the models of space-time cube and multivariable space-time cube are the effective approaches to visualizing movement data.

\section{REFERENCES}

[1] D. J. Peuquet, "It's About Time: A conceptual framework for the representation of temporal dynamics in geographic information systems," Annals of the Association of American Geographers, Taylor \& Francis, vol. 84, no. 3, pp. 441-461, 1994.

[2] G. Andrienko, N. Andrienko, P. Bak, D. Keim, S. Kisilevich, and S. Wrobel, "A conceptual framework and taxonomy of techniques for analyzing movement," Journal of Visual Languages and Computing, vol. 22, pp. 213-232, 2011.

[3] N. Andrienko, G. Andrienko, N. Pelekis, and S. Spaccapietra, "Basic concepts of movement data," Data Mining and Privacy, Geographic Knowledge Discovery, Springer, 2008, pp.15-38.

[4] M. Friendly, "Visions and re-visions of Charles Joseph Minard," Journal of Educational and Behavioral Statistics, vol. 27, no. 1, pp. 31-51, 2002.

[5] M. J. Kraak, Mapping Time, Illustrated by Minard's map of Napoleon's Russian Campaign of 1812, ESRI Press, 2014.

[6] T. Hagerstrand, "What about people in regional science?" in Proc. Papers of Ninth European Congress of Regional Science Association, vol. 24, 1970, pp. 7-21.

[7] M. J. Kraak. "Geovisualization illustrated," ISPRS Journal of Photogrammetry \& Remote sensing, vol. 57, pp. 390-399, 2003.

[8] T. N. Hong, T. K. D. Chi, T. B. Tha, and V. T. Phuoc, "Visualization of spatio-temporal data of bus trips," in Proc. the IEEE 2012 International Conference on Control, Automation and Information Science, November 26-29, 2012, Hochiminh City, Vietnam.

[9] V. T. Tran, V. P. Tran, and T. T. Bui, "Representing uncertain time on space-time cube for bus movement," in Proc. ICCAIS 2013 Conference, IEEE 2013 International Conference on Control, Automation and Information Science, November 25-28, 2013, Nha Trang, Vietnam.

[10] M. J. Kraak, "The space-time cube revisited from a geovisualization perspective," in Proc. the 21 st International Cartographic Conference (ICC) "Cartographic Renaissance", 2003, pp. 1988-1996.

[11] N. Andrienko and G. Andrienko, "Spatial generalization and aggregation of massive movement data," IEEE Transactions on visualization and computer graphics, vol. 17, no. 2, pp. 205-219, 2011.

[12] V. P. Tran and T. H. Nguyen, "Multivariate-space-time cube to visualize multivariate data," International Journal of Geoinformatics, vol. 8, no. 4, pp. 67-74, 2012.

[13] X. Li and M. J. Kraak, New Views on Multivariable Spatio-temporal Data: the Space-time Cube Expanded, 2005.

[14] V. P. Tran and T. H. Nguyen, "Visualization cube for tracking moving object," in Proc. the Computer Science and Information Technology, Information and Electronics Engineering, IACSIT Press, vol. 6, 2011 , pp. 258-262.

[15] V. T. Phuoc, T. N. Hong, V. T. Trung, and T. B. Tha, "On an approach to visualizing data of flying objects," in Proc. the IEEE 2014 International Conference on Control, Automation and Information Science (ICCAIS 2014), Gwangju, Korea, December 2-5, 2014.

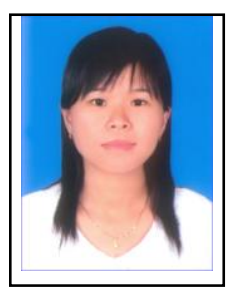

Hong Nguyen Thi is a lecturer of Rubber Industrial College in Binhphuoc Province, Vietnam. She received M.S in computer science from Vietnam National University - HCMC in 2009. She is a Ph.D. student of the UIT with the topic of multivariate data visualization in GIS and has presented some researches at Some international conferences and journal. 